\title{
De-hospitalization of the pediatric day surgery by means of a freestanding surgery center: pilot study in the lazio region
}

\author{
Giovanni Mangia ${ }^{1 *}$, Franco Bianco ${ }^{1}$, Alma Ciaschi ${ }^{2}$, Elisabetta Di Caro ${ }^{1}$, Eufrasia Frattarelli ${ }^{1}$ and \\ Giacinto Antonio Marrocco ${ }^{3}$
}

\begin{abstract}
Background: Day surgery should take place in appropriate organizational settings. In the presence of high volumes, the organizational models of the Lazio Region are represented by either Day Surgery Units within continuous-cycle hospitals or day-cycle Day Surgery Centers. This pilot study presents the regional volumes provided in 2010 and the additional volumes that could be provided based on the best performance criterion with a view to suggesting the setting up of a regional Freestanding Center of Pediatric Day Surgery.

Methods: This is an observational retrospective study. The activity volumes have been assessed by means of a DRG (Diagnosis Related Group)-specific indicator that measures the ratio of outpatients to the total number of treated patients (freestanding indicator, FI). The included DRGs had an FI exceeding the $3^{\text {rd }}$ quartile present in at least a health-care facility and a volume exceeding $0.5 \%$ of the total patients of the pediatric surgery and urology facilities of the Lazio Region. The relevant data have been provided by the Public Health Agency and relate to 2010. The best performance FI has been used to calculate the theoretical volume of transferability of the remaining facilities into freestanding surgery centers. Patients under six months of age and DRGs common to other disciplines have been excluded. The Chi Square test has been used to compare the Fl of the health-care facilities and the $\mathrm{Fl}$ of the places of origin of the patients.

Results: The DRG provided in 2010 amounted to a total of 5768 belonging to 121 types of procedures. The application of the criteria of inclusion have led to the selection of seven final DRG categories of minor surgery amounting to 3522 cases. Out of this total number, there were 2828 outpatients and 694 inpatients. The recourse of the best performance determines a potential transfer of 497 cases. The total outpatient volume is $57 \%$. The Chi Square test has pointed to a statistically significant difference of the facilities and to a non-significant difference of inferiority of the regional places of origin with respect to the city of Rome.
\end{abstract}

Conclusions: The activity volumes would seem to support the setting up of a Freestanding Regional Center of Pediatric Day Surgery. This Center represents the healthcare facility that is most likely to allow a de-hospitalization process. Subsequent studies will be required to confirm the validity of this pilot study.

\section{Introduction}

The selection of both the type of admission, whether regular or in a day surgery unit, and the continuouscycle or exclusively daytime facility represents a fundamental aspect in the organization of minor surgery for pediatric patients.

\footnotetext{
* Correspondence: mangia.giovanni@fastwebnet.it

'Departement of Anesthesia, San Camillo Hospital, Rome, Italy

Full list of author information is available at the end of the article
}

The regular admission should be restricted to patients whose age and clinical conditions contraindicate the recourse to day surgery [1]. The healthcare arrangements are extremely different.

The Lazio Region has identified three basic types of healthcare facilities based on a State-Regions agreement $[2,3]$. Single or multi-specialist Integrated Day Surgery Unit, located within a continuous cycle hospital facility devoted to day care. This type of facility may be likened

\section{() Biomed Central}

(c) 2012 Mangia et al; licensee BioMed Central Ltd. This is an Open Access article distributed under the terms of the Creative Commons Attribution License (http://creativecommons.org/licenses/by/2.0), which permits unrestricted use, distribution, and reproduction in any medium, provided the original work is properly cited. 
to the model of the Hospital-based facility of the AngloSaxon countries [4]. Day Surgery Center, involving a facility exclusively devoted to day-care admissions. Dedicated nursing beds within a regular hospital unit when the activity is sporadic. Similar settings are also present in other European countries [5].

Both in Italy and in Europe, the "Day Surgery Centers" particularly in the pediatric sector - have failed to become as widespread as in the United States of America [US], where, defined Ambulatory Surgery Centers (ASC) or Pediatric Outpatient Pediatric Surgery (POPS), they have increased to a considerable extent. Being these facilities autonomous with respect to hospitals, they are also defined as freestanding surgery centers [6,7].

The purpose of this study is to establish, having recourse to an activity indicator, the regional healthcare volumes provided through day surgery centers and the healthcare volumes provided though regular hospitalization that could be transferred to day surgery centers based on the healthcare facility best performance criterion. The indicator that has been devised (freestanding indicator, FI) measures the outpatient/total patient ratio of the most frequent minor surgery DRGs (Diagnosis Related Groups) handled in 2010 by the pediatric surgery facilities in the Lazio Region.

We believe that the resulting data are likely to prove useful to decision makers for planning subsequent economic and marketing studies, verifying the hypothesis that the setting up of a freestanding inter-hospital pediatric day surgery center may allow the de-hospitalization of most pediatric surgical activities.

\section{Materials and methods}

This preliminary observational cohort study has not required the opinion of the ethical committee nor any informed consent being a population study.

The STROBE-Statement checklist [8] has been taken into consideration for its draft. The study has been conducted in the Lazio Region and the data being dealt with relates to the DRGs produced in 2010 by the regional surgery and pediatric urology facilities. The data were provided on line by the Public Health Agency of the Lazio Region (ASP Lazio).

Outpatients and inpatients of the individual DRGs have been evaluated based on the healthcare providers and the patients' places of origin. The healthcare facilities were the Operating Units (UO) of pediatric surgery and urology of the Lazio Region: Pediatric Hospital "Bambin Gesù" (OPBG, with two separate branches), "San Camillo" Hospital. (AOSCF), "A. Gemelli" General Hospital, "Umberto I" General Hospital. These facilities are located within the city of Rome, with the exception of a branch of the "Bambin Gesù" hospital which is located in the northern area of the Rome Province. The organizational models adopted by these facilities have been studied by means of separate surveys. The study has also taken into consideration the DRGs produced by non-specialized pediatric facilities. The places of origin that have been taken into consideration have been Rome and the Rome Province, Latina and the Latina Province, Frosinone and the Frosinone Province, Viterbo and the Viterbo Province, Rieti and the Rieti Province, as well as cities and Provinces outside the Lazio Region.

A selection of the DRGs has been made having recourse to the FI, a DRG-specific indicator that measures the ratio of outpatients to the total number of treated patients. The numerator is represented by the patients discharged on the same day of treatment; the denominator is represented by the sum of all the treated patients of the DRG under consideration. Therefore, the denominator includes regular one-day admissions (one-day surgery) and admissions for 2 or more days. Assessing the level of elimination of regular hospital admissions and, therefore, of overnight stays, this index points to the percentage of services that may be theoretically provided by freestanding centers.

The criteria for inclusion into the study (with a ranking process) were all the DRGs with an FI exceeding the $3^{\text {rd }}$ quartile present in at least a providing facility, with volumes of outpatients exceeding $20 \%$ and a volume of cases exceeding the entire case study by $0.5 \%$. The highest value of the indicator identifies the best performing facility. The criteria for exclusion were all the patients under six months of age and all the DRGs in common with other disciplines.

The DRG-specific FI of the best performing providing facility has allowed calculating the theoretical volume of transferability in freestanding mode of the remaining facilities. The FI was also calculated based on the place of origin of the patients.

The statistical analysis had recourse to the Chi Square test for comparing the FI of the providing facilities and of the various places of origin. The study sample size has been the population being considered, that is 5768 cases. 361 cases would have been enough to calculate the size of a hypothetical random sample for a $95 \%$ confidence interval. The power calculated for the test was $80 \%$ with a level of significance of $5 \%$. The statistical analysis was carried out with OpenEpi open-source statistic software (Copyright__ 2003, 2008 Andrew G. Dean and Kevin Mt. Sullivan, Atlanta, GA, USA).

With reference to inpatients, the prevalence of a onenight hospital admission (one-day surgery) was evaluated with respect to the admissions for two or more nights. An unspecified number of patients experienced during their hospitalization a change in their planned admission from outpatient to inpatient and vice versa. 


\section{Results}

The DRGs provided in 2010 by the pediatric surgery and urology facilities, with the exclusion of patients under six months of age, amounted to a total of 5768, belonging to 121 types of procedures (Figure 1, flowchart).

The exclusion of DRGs shared with disciplines other than that taken into consideration or with a better performance FI under the $3^{\text {rd }}$ quartile or with volumes of outpatients under $20 \%$ or DRGs with volumes under $0.5 \%$ of the total case study, has led to the selection of seven final DRG categories of minor surgery amounting to a total of 3522 cases. Out of these, 2828 - equal to $49 \%$ of the initial case study - were outpatients, while 694 were inpatients. The weighted average of the FI by DRG typology, corresponding to outpatients, is $81 \%$ (Table 1)

The application of the best performance criterion has determined a potential transfer of 497 additional cases belonging to the seven selected DRGs, equal to $14.1 \%$. Adding the real volumes to the transferable volumes, it turned out that the total DRGs were 3325. The latter represent $57.5 \%$ of the outpatients with respect to the original DRGs, and 95\% of the selected final DRGs (Table 2).

The organizational setup of the pediatric surgery and urology facilities are extremely heterogeneous. Out of the 2828 outpatients, 2825 have been treated in two facilities with an FI of 86.6 and 94.3 , respectively. The comparison with the Chi Square statistics of the data relative to these two facilities is highly significant $(\mathrm{p}<$ 0.001 ) from a statistical viewpoint. The ratio of the seven selected DRGs to the total services provided by the healthcare facilities ranges between 40 and $72 \%$ (Additional file 1).

The FI calculated based on the places of origin of the patients is quite homogeneous. The Chi Square comparison of outpatients and inpatients between Rome and the others cities and Provinces in the Lazio Region, with the exclusion of Latina and the Latina Province, has not provided statistically significant differences of superiority ( $p>0.05)$. The comparison between Latina and the Latina Province and Rome is statistically significant and the FI is higher for Latina and the Latina Province. The FI for the areas outside the Region is 70.5; the Chi Square comparison between patients from the Lazio Region and those from outside the said Region is highly significant $(\mathrm{p}<0.001)$ from a statistical point of view (Table 3). With reference to the inpatients, the one-day surgery accounts for $33.1 \%$ with respect to the DRG being considered.

\section{Discussion}

The application of the exclusion criteria to the initial case study has led to the selection of seven types of

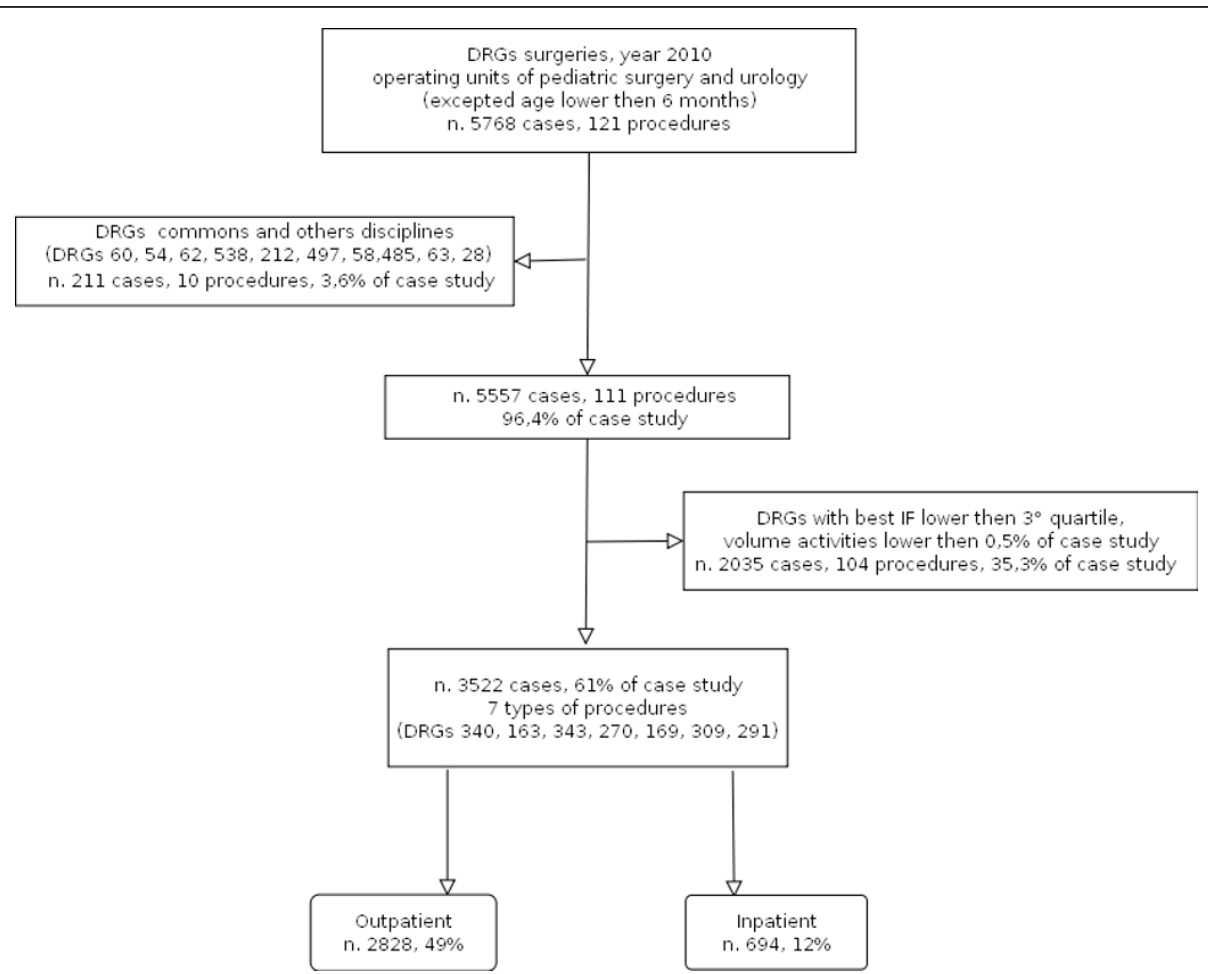

Figure 1 Flowchart DRGs produced in 2010 by the regional surgery and pediatric urology facilities. 
Table 1 Index of selected Freestanding Drg and volumes of activity.

\begin{tabular}{llccccc}
\hline DRG & description & Outpatient & Inpatient & $\begin{array}{c}\text { Freestanding index } \\
\text { (weighted avg) }\end{array}$ & $\begin{array}{c}\text { Outpatient/opening drg } \\
\%\end{array}$ & $\begin{array}{c}\text { Outpatient/closing drg } \\
\%\end{array}$ \\
\hline 340 & Testicular surgery & 1337 & 345 & 79.5 & 23.2 & 37.9 \\
163 & Hernia surgery & 647 & 140 & 82.2 & 11.2 & 18.4 \\
343 & Circumcision & 633 & 94 & 87.1 & 11 & 18 \\
270 & Other skin, etc. surgery & 114 & 43 & 72.6 & 2 & 3.3 \\
169 & Mouth, etc. surgery & 50 & 13 & 79.4 & 0.9 & 1.4 \\
309 & Minor bladder surgery & 32 & 22 & 59.2 & 0.5 & 0.90 \\
291 & Thyroglossal duct surgery & 15 & 37 & 28.8 & 49 & 0.4 \\
& & $2828^{*}$ & $694^{*}$ & 81 & 40.3 \\
\hline
\end{tabular}

Data relating to all facilities.

* sum of all facilities

procedures that represent $61 \%$ of the total DRGs provided by the pediatric surgery and urology facilities of the Lazio Region in 2010. In these DRGs, $80 \%$ are outpatients. The best DRG-specific performance determines a theoretical $10 \%$ increase. There are no sufficient clinical and organizational data to determine the causes of the high recourse to the one-day surgery, which accounts for nearly $1 / 3$ of all regular admissions. It is quite likely that even a part of these admissions with overnight stay could be transferred to day surgery.

The age group included in the study is represented by patients who are over six months of age. This helps to assess the regional volume of procedures that could be supposedly carried out in a freestanding surgery center using the same minimum age cut-off for treatment of the Ambulatory Surgery Centers [1]. As for patients under six months of age, the literature concurs that newborn babies and premature babies with a gestational age below the $60^{\text {th }}$ week of post-conceptional life [9] should be excluded from day surgery. It is advisable that, depending on the protocols adopted by the individual healthcare facilities, babies whose age is included between two and six months be admitted to a hospitalbased day surgery unit or dedicated nursing beds [1].
With reference to the age group referred to above, a total of 588 patients have been treated in 2010 by the healthcare facilities taken into consideration. Most of them have been treated having recourse to a regular admission procedure and, in fact, the FI is very low (14.6\%).

The organizational models and the FI of the healthcare facilities are independent. Paradoxically, the model of partial de-hospitalization, represented by day surgery units, features a lower FI than the model of the dedicated nursing beds in departments for acute patients. The difference is statistically significant. The analysis of the causes is complex and the phenomenon highlights the inadequacy of the FI as the only indicator of the quality and appropriateness of the day surgery services. It is quite likely that an improved understanding of these causes could be gained by monitoring the indicators laid down in the regulations of the Lazio Region [2]. As for the latter, the one relative to the measures connected with the dedicated nursing beds model could be an aspect to be kept under observation. Other elements that should be considered include the analysis of costs in the two models and the time analysis of the activity data. In any case, it may be assumed that, given the want of

Table 2 Best performance.

\begin{tabular}{|c|c|c|c|c|c|c|}
\hline DRG & Description & $\begin{array}{l}\text { Outpatient: best } \\
\text { performance (\%) }\end{array}$ & $\begin{array}{c}\text { Outpatient: } \\
\text { transferable ** }\end{array}$ & $\begin{array}{c}\text { Outpatient } \\
\text { total }\end{array}$ & $\begin{array}{c}\text { Outpatient/operning } \\
\text { drg }\end{array}$ & $\begin{array}{c}\text { Outpatient/closing } \\
\text { drg }\end{array}$ \\
\hline 340 & Testicular surgery & 94.3 & 249 & 1586 & 27.5 & 45 \\
\hline 163 & Hernia surgery & 91.9 & 76 & 723 & 12.5 & 20.5 \\
\hline 343 & Circumcision & 97.7 & 78 & 711 & 12.3 & 20.9 \\
\hline 270 & $\begin{array}{l}\text { Other skin, etc. } \\
\text { surgery }\end{array}$ & 93.7 & 34 & 148 & 2.6 & 4.2 \\
\hline 169 & Mouth, etc. surgery & 93.3 & 9 & 59 & 1 & 1.7 \\
\hline 309 & $\begin{array}{l}\text { Minor bladder } \\
\text { surgery }\end{array}$ & 91.4 & 17 & 49 & 0.8 & 1.4 \\
\hline \multirow[t]{2}{*}{291} & Thyroglossal duct & 91.6 & 34 & 49 & 0.8 & 1.4 \\
\hline & & 94.4 weighted & 497 & 3325 & 57.5 & 95.1 \\
\hline
\end{tabular}


Table 3 Geographical origin of patients.

\begin{tabular}{cccccc}
\hline Origin & Outpatient & Inpatient & IF & Test Chi-square (City of Rome) & p \\
\hline Rome - City & 1302 & 317 & 80.4 & - & - \\
Rome - Province & 694 & 139 & 83.3 & 8.853 & 6.459 \\
Latina and its province & 194 & 27 & 7.7 & 0.481 & 0.091 \\
Frosinone and its province & 149 & 42 & 78 & 2.211 & 0.011 \\
Viterbo and its province & 92 & 14 & 86.8 & 0.004 & 0.137 \\
Rieti and its province & 59 & 141 & 80.8 & 20.469 & 0.947 \\
Other provinces & 338 & 694 & $80.3^{*}$ & & 0.000 \\
\end{tabular}

* weighted average

resources, in a forthcoming future the model of dedicated nursing beds in mixed (adult and pediatric) continuouscycle hospitals could be negatively affected by the progressive greater care complexity of adult patients with respect to pediatric patients. This phenomenon could be heightened when the surgical activity takes place in operating units that are not reserved to day surgery. On the other hand, the obstacles to the development of day surgeries could be less significant in exclusively pediatric hospitals owing to the lower ASA Physical Status grading values and the relative time stability of such conditions with respect to grown-ups. Furthermore, with a view to ensuring ongoing quality, effectiveness and efficiency, the only pediatric hospital - the Bambin Gesù Pediatric Hospital (OPBG) - got prevailingly organized with day surgery units for the patients' stay and specific operating units.

The place of origin of the patients, often considered a factor limiting the recourse to a day surgery if located far away from the healthcare facility, has not conditioned the choice of the type of hospital admission within the Region. This may be inferred from the comparison of the FI with the Chi Square test, which has not proved significant based on the place of origin. Indeed, the data relative to Latina and its Province point to the inferiority of Rome. The case is altogether different when dealing with places of origin from outside the Region, the significance of which gives evidence of an inappropriate use of the hospital as the place of an overnight stay.

The study presents additional limitations.

The FI has been constructed by modifying the indicators used for the assessment of the appropriateness of hospital admissions of the APPRO (Appropriateness of hospital admissions) method [10], the transferability of the volumes of day surgery of the MAAP (Model of Analysis of the Procedure Appropriateness) method [11] and the assessment of the balanced scorecard system performance [12,13].

Until now, such methodologies have been prevailingly used for adult patients.
The APPRO, built by the Public Health Agency of the Lazio Region, is based on hospital discharge cards (SDO) and has partly recourse to an isogravity classification system called All Patient Refined Diagnosis Related Groups (APR-DRGs) [14]. The method has been conceived with a view to assessing the behavior of hospital facilities in the provision of care services characterized by a low complexity to patients who are not affected by a clinically severe illness, reasonably assuming that, in these cases, the regular admission to a hospital is as a rule an inappropriate organizational procedure [15]. The APPRO method is used in particular to calculate the admissibility thresholds (to regular admission) for DRGs with a high risk of inappropriateness [16]. In this method, the DRG-specific hospital or regional threshold of "tolerated inappropriateness" is a proportion where the numerator is the number of regular admissions of patients affected by illnesses with a minimum level of severity $>1$ day and the denominator is the total number of regular admissions of patients affected by illnesses with a minimum level of severity to the hospital or the day surgery unit. The APPRO method is used in order to enforce systems of rewards or sanctions.

The MAAP method has been adopted by the Puglia Region to build indicators of the care setting transferability, DRGs with a high volume of cases, from regular hospitals admissions to day hospitals and ambulatory surgery centers. The transferability volume indicator is used when the DRG volume exceeds $0.5 \%$ of the admissions for the entire case study.

The assessment of the performance of the healthcare systems has been made on behalf of the Ministry of Health by the Management and Health Laboratory of the Sant'Anna School of Advanced Studies in Pisa through a set of 34 indicators calculated on the SDOs in 2007 and 2008 [17]. The regional health systems were benchmarked having recourse to target and quintiles methods that allow moving from measurement to assessment. These indicators include those that analyze the appropriateness of the surgical services, namely the share of surgical DRGs falling within the essential levels of care (LEAs), provided in day 
surgery and one-day surgery facilities. The volume of transferability into freestanding centers of the regular admissions of the least performing facilities with respect to the best performance that has been adopted in this study presents a few analogies with the assessment systems referred to above, with the difference that the method of quintiles has been turned into quartiles.

The available data are incomplete and this has not allowed determining the extent of the change of the system of admission from outpatient to inpatient and vice versa. This aspect should be monitored as it could invalidate the day surgery activity. Hence, the assessment of the healthcare results with administrative data has both advantages and disadvantages. The immediate access to data on relatively uniform computer files and the possibility of estimating shares of inappropriateness within the context of selected case records are definite advantages. On the other hand, the disadvantages are due to the uncertainty of the nature of the administrative datum (potentially incomplete, inaccurate, and distorted) and the absence of references to the context where the admission takes place (e.g., social conditions of the patient). It ensues that the inner validity of the appropriateness assessment methods depends on the comprehensiveness and accuracy of the SDO compilation and coding.

Notwithstanding the aforementioned limitations, there are also additional systems that back up the recourse to administrative data. In fact, the FI is similar to the ASC proportion of the pay for performance (P4P) system [18]. Within this context, it is used in a few states of the USA in both adult and pediatric age starting from patients over the sixth month of life. The P4P system produces a rewarding payment mechanism for the best performing facilities $[19,20]$.

The avoidable hospitalization is a subject that is well known to pediatricians. By analogy with the studies of the ambulatory care-sensitive conditions that have led to a radical reduction of the medical admissions to hospitals, a few studies that are likely to promote a partial surgical de-hospitalization should be implemented [21]. In fact, in the surgical field, the comparison between ASC and hospital-based facility is still an open question, besides being quite a controversial point [22-24].

Furthermore, there is also considerable confusion about the meaning of de-hospitalization [25]. For administrative personnel, de-hospitalization merely means the passage of a few types of services from the regular hospital setup to a day surgery unit, regardless of the care setting where the process takes place. On the other hand, one needs considering the type of hospital wards and surgery units and, in particular, their inclusion in continuous cycle or day-only facilities. In our opinion, the real de-hospitalization of day surgery is represented by the provision of that activity in daytime facilities and, therefore, with specific hospital wards and surgery units.

The day surgery cases reported in this pilot study only concern pediatric surgery and urology. They should be considered minimum case studies susceptible of increase, since other specialties are also having recourse to day surgery (e.g., ORL, orthopedics, ophthalmology, digestive endoscopy, etc.). Therefore, a considerable part of the services currently provided in a number of continuous-cycle hospital facilities could be concentrated in a hypothetical inter-hospital health facility. We believe that the positive trend of the outpatient/inpatient ratio with respect to previous years, the significant volumes of real and potential activities highlighted in this pilot study, the abolition of the one-day surgery and the extension of the analysis to other disciplines could further the setting up of a freestanding inter-hospital facility of pediatric day surgery in the Lazio Region.

\section{Conclusions}

The implementation of a healthcare facility does not depend solely on the total activity volumes, even though this is an important aspect. This pilot study could turn into the starting point of subsequent studies focusing on health management (e.g., cost analysis), on the expression of the parents' preferences (e.g., conjoint analysis), on marketing, etc., with a view to evaluating the feasibility of a de-hospitalization of the pediatric day surgery through a freestanding surgery center in a Region where the existing facilities are found within hospitals characterized by a high healthcare complexity and are concentrated within the city of Rome.

\section{Conflicts of interest}

The authors declare that they have no competing interests.

\section{Additional material}

Additional file 1: Organisational models of the Child Surgery Operational Units.

\section{Acknowledgements}

We wish to thank Dr. Laura Cacciani, Performance Evaluation Coordinator of the Public Health Agency of the Lazio Region (ASP Lazio).

\section{Author details}

${ }^{1}$ Departement of Anesthesia, San Camillo Hospital, Rome, Italy. ${ }^{2}$ Departement Production Flow, Local Health Rome A, Italy. ${ }^{3}$ Department Pediatric Surgery, San Camillo Hospital, Rome, Italy.

\section{Authors' contributions}

All authors were involved in the conception of the study. GM carried out data extraction. He is the corresponding author of the paper. GM conducted 
statistical analysis. GM drafted the paper with contributions from the coauthors. All authors read and approved the final manuscript prior to submission.

Received: 25 November 2011 Accepted: 1 February 2012

Published: 1 February 2012

\section{References}

1. Fishkin S, Litman RS: Current issues in pediatric ambulatory anesthesia. Anesthesiol Clin North America 2003, 21(2):305-11.

2. Regional Board of Lazio: Recognition of the agreement between the Ministry of Health, the Regions and the Autonomous Provinces of Trent and Bozen to approve the guidelines governing the surgical activities that may be carried in day surgery units. Resolution no. 423 of March 25, 2005., Available from URL: http://www.asl.vt.it/Staff/Sistemilnformativi/ Documentazione/sio/pdf/DGR423-2005.pdf.

3. Sommella L: I contesti di erogazione delle prestazioni chirurgiche. In || reparto operatorio. Progettazione, organizzazione, controllo. Edited by: Cambieri A, Girardi F, D'Alfonso ME, Luongo AM, Bock M. Roma: Il Pensiero Scientifico Editore; 2010:437-468.

4. Al-Amin M, Housman M: Ambulatory surgery center and general hospital competition: entry decisions and strategic choices. Health Care Manage Rev 2011.

5. International Association for Ambulatory Surgery: Policy Brief, Day Surgery Development and Practice, Available from URL: http://www.iaas-med.com/ index.php/iaas-initiatives.

6. Hedley-Whyte J, Milamed DR: The evolution of site of surgery. U/ster Med J 2006, 75(1):46-53.

7. Rabbitts JA, Groenewald CB, Moriarty JP, Flick R: Epidemiology of ambulatory anesthesia for children in the United States: 2006 and 1996. Anesth Analg 2010, 111(4):1011-5, Available from URL: http://www. anesthesia-analgesia.org/content/111/4/1011.full.pdf+html.

8. Von Elm E, Altman DG, Egger M, Pocock SJ, Gøtzsche PC, Vandenbroucke JP, STROBE Initiative: Strengthening the Reporting of Observational Studies in Epidemiology (STROBE) statement: guidelines for reporting observational studies. Epidemiology 2007, 18(6):800-4, Available from URL: http://www.strobe-statement.org/index.php?id=strobehome.

9. Bettelli G: High risk patients in day surgery. Minerva Anestesiol 2009, 75(5):259-68.

10. Di Domenicantonio R, Filocamo A, Baglio G, Canonaco D, Conte A, Jozdani SM, Maurici M, Prota F, Materia E, Guasticchi G: Evaluating hospital appropriateness with different tools: administrative data versus analytic review. Ann Ig 2004, 16(1-2):79-94, Italian.

11. Regional Board of Puglia: Approval of the "Model of analysis of the organizational appropriateness of hospital admission by list of procedures" (M.A.A.P.). Resolution no. 834 of May 27, 2008., Available from URL: http://www.regione.puglia.it/web/files/sanita/appropriatezza.pdf.

12. Nuti S: II sistema di valutazione delle aziende sanitarie toscane. In II bilanced Scorecard nelle aziende sanitarie. Edited by: Baraldi S. Milano: Franco Angeli; 2005:

13. Nuti S, Bonini A, Murante AM, et al: Performance assessment in the maternity pathway in Tuscany region. Health Serv Manage Res 2009, 22(3):115-21.

14. Fantini MP, Cisbani L, Manzoli L, Vertrees J, Lorenzoni L: On the use of administrative databases to support planning activities: the case of the evaluation of neonatal case-mix in the Emilia-Romagna region using DRG and APR-DRG classification systems. Eur J Public Health 2003, 13(2):138-45

15. Baglio G, Di Domenicantonio R, Materia E, Guasticchi G: La valutazione dell'appropriatezza organizzativa per modulare le tariffe della compensazione interregionale della mobilità sanitaria. Tendenze nuove 2003, 2:107-120.

16. Di Domenicantonio R, Lorenzoni L, Lispi L, Guasticchi G, Materia E: Aggiornamento del metodo APPRO per valutare l'appropriatezza organizzativa con dati amministrativi. Tendenza nuove 2008, 2:151-160

17. Nuti S: La valutazione della performance in sanità. Bologna: II Mulino; 2008.

18. Appropriate resource use measures for P4P MY. 2009 [http://www.ha. org/pdfs_documents/related_resources/ARUMeasures.pdf].
19. Mannion R, Davies HT: Payment for performance in health care. BMJ 2008, 336(7639):306-8, PubMed PMID: 18258966; PubMed Central PMCID: PMC2234517.

20. Castaldi S, Bodina A, Bevilacqua L, Parravicini E, Bertuzzi M, Auxilia F: Payment for performance (P4P): any future in Italy? BMC Public Health 2011, 11:377.

21. Parker JD, Schoendorf KC: Variation in hospital discharges for ambulatory care-sensitive conditions among children. Pediatrics 2000, 106(4 Suppl):942-8.

22. Chukmaitov AS, Menachemi N, Brown S, Saunders C, Brooks RG: A comparative study of quality outcomes in freestanding ambulatory surgery centers and hospital-based outpatient departments. Health Services Research 2008, 43:1485-1504[http://www.ncbi.nlm.nih.gov/pmc/ articles/PMC2653888/].

23. Grisel J, Arimand E: Comparing quality at an ambulatory surgery center and a hospital-based facility: preliminary findings. Otolaryngol Head Neck Surg 2009, 141(6):701-9.

24. Trentman TL, Mueller JT, Gray RJ, Pockaj BA, Simula DV: Outpatient surgery performed in an ambulatory surgery center versus a hospital: comparison of perioperative time intervals. Am J Surg 2010, 200(1):64-7.

25. Zocchetti C, Cislaghi C: Measuring de-hospitalization is not an easy job. Pay attention! Epidemiol Prev 2011, 35(1):61-2.

doi:10.1186/1824-7288-38-5

Cite this article as: Mangia et al:: De-hospitalization of the pediatric day surgery by means of a freestanding surgery center: pilot study in the lazio region. Italian Journal of Pediatrics 2012 38:5.

\section{Submit your next manuscript to BioMed Central and take full advantage of:}

- Convenient online submission

- Thorough peer review

- No space constraints or color figure charges

- Immediate publication on acceptance

- Inclusion in PubMed, CAS, Scopus and Google Scholar

- Research which is freely available for redistribution

Submit your manuscript at www.biomedcentral.com/submit
Ciomed Central 